W. Stoeder, Alkalö̈bostimmung der Bolivia-Chinarinden etc. 243

und die Hausfrauen finden in dieser Einrichtung ein Analogon ihrer Frucht- und Gemüse-Conserven.

Das Einstopfen der Vegetabilien in die Blechbüchse ist zwar ein wenig mühsam, diese Mühe belohnt sich aber zehnfach in der Ersparniss an Aerger und Zeitverlust wegen Verderbens und Schimmelns, und sogar pecuniär durch die verminderte Ergänzung selbst der narkotischen Vegetabilien.

\title{
Alkaloïdbestimmung der Bolivia-Chinarinden auf der internationalen Gartenbauausstellung in Amsterdam.
}

Von Professor W. Stoeder in Amsterdam. ${ }^{1}$

Auf der im Jahre 1877 zu Amsterdam abgehaltenen internationalen Gartenbauausstellung bildete die Chinaausstellung einen wichtigen und im hohen Grade interessanten Theil. Neben den Producten der javanischen Chinacultur erschien in dieser Abtheilung von besonderem Werthe eine Sammlung bolivianischer Chinarinden von dem niederländischen Consul E. Schuhkraft herrührend und so vollständig, wie kaum jemals eine derartige Collection gewesen, von der merkwürdigen Monopol - China an, deren Mutterpflanze längst ausgerottet, aber höchst wahrscheinlich noch von Weddell (1846) und Markham (1859) bewundert wurde, bis zu den ganz werthlosen Rinden herab, welche die eingeborenen Chinasammler (Cascarilleros) betrügerischer Weise unter die guten Sorten mengen, unter 27 Nummern in anschaulicher Weise gruppirt. Durch die Bemühungen Schuhkraft's, dessen Verdienst die Jury durch Ertheilung der silbernen Medaille mit Recht ehrte, ist es möglich geworden, zum ersten Male eine Alkaloïdbestimmung in Calisayarinden auszuführen, deren Ursprung von einer bestimmten Kindenspecies festgestellt worden ist, oder deren Abstammung doch mit grösserer Sicherheit bekannt ist und an Ort und Stelle festgestellt

1) Aus Haaxmann's Nieuw Tijdschrift roor do Pharmacie in Nederland mitgetheilt von Th. Husemann. 
wurde. Die bisher ausgeführten derartigen Analysen haben für die Sorte insofern wenig Werth, als die im Handel vorkommende Calisaya-China in der Regel einen gemengten Handelsartikel darstellt, dessen Abstammung und Ursprung nicht mit Sicherheit zu ermitteln ist. Die durch die wohlwollende Vermittelung von Professor C. A. J. A. Oudemans und Dr. H. F. R. H u brecht erhaltenen Proben waren grösstentheils Fragmente längerer Stücken und Röhren und reprïsentirten desshalb sehr gut die zu untersuchende China.

Was die bei der Analyse befolgte Methode anlangt, so wurden für jede Alkaloïdbestimmung $25 \mathrm{~g}$. pulverisirte Chinarinde mit $10 \mathrm{~g}$. Calciumhydroxyd und $60 \mathrm{~g}$. Wasser in der Weise gemengt, dass zuerst der Kalk in dem Wasser sorgfältig vertheilt und dann das Chinapulver zugesetzt wurde. Der erhaltene homogene Brei wurde stets mindestens 24 Std., während welcher Zeit wiederholtes Umrühren stattfand, bei Seite gestellt und dann erst anf dem Wasserbade durch gelindes Erwärmen vollkommen ausgetrocknet. Das fein ver. riebene, vollkommen homogene Pulver wurde nun in einen zur Ausführung der betreffenden Analysen besonders geeigneten Deplacirungs - Destillations - Apparat gebracht, welcher es ermöglicht, das zeitraubende und lästige Auskochen des Chinakalkes mit Spiritus nach De Vrij und Moens zu vermeiden und der eine Modification der früher von Payen, Ma umené, Cazeneure und Caillol angegebenen Apparate darstellt. Als Deplacirungsflüssigkeit wurde $92 \%$ Spiritus, welcher $20 \%$ Chloroform enthielt, benutzt, eine Mischung, die sich ganz vorzüglich nicht allein wegen ihres besonders hohen Lösungsvermögens für Chinaalkalö̈de hierzu eignet, sondern auch weil sie die Alkaloïde in weit reinerem Zustande aufnimmt, als der unverdünnte Alkohol dies thut. Der betreffende Apparat, welcher von Steinhoff in ausgezeichneter Weise aus Glas angefertigt wird, hat den grossen Vortheil, dass er Stunden, ja Tage lang ohne besondere Mühe in Gang gehalten werden kann und dass zum Ausziehen nur eine sehr rreringe Flüssigkeitsmenge nöthig ist, so dass für jede Chinaprobe nur $100 \mathrm{~g}$. der angegebenen Mischung von Alkohol 
und Chloroform nothwendig sind. Auf eine genaue Beschreibung des fraglichen Apparats muss hier verzichtet werden und sei nur erwähnt, dass bei Bearbeitung von $25 \mathrm{~g}$. Chinapulver von mittlerem Alkaloïdgehalt der Apparat ca. 6 Std. im Gange erhalten werden muss. Nachdem die Operation vollendet ist und nach geschehener Abkühlung überzeugt man sich, ob alle Chinaalkaloïde ausgezogen seien. Es geschieht dies einfach in der Weise, dass man $2-3$ Tropfen der noch an dem Chinakalk haftenden Deplacirungsflüssigkeit auf einem Uhrglase sammelt und an der Luft oder auf dem Wasserbade verdunsten lässt, dann den zurückbleibenden Fleck von gelöstem Kalk in einem Tropfen verdünnter Salzsüure auflöst. Entsteht in dieser Lösung durch Znsatz von Kaliumkadmiumjodid keine Trübung, so kann man sicher sein, dass der Chinakalk kein Alkaloïd mehr enthält. Hat diese Probe die vollkommene Extraction dargethan, so giesst man $50 \mathrm{Ccm}$. Wasser auf den noch in dem Deplacirungsrohre verbleibenden Chinakalk, welche Quantität vollständig genügt, um die noch an dem Kalke haftende Deplacirungsfliussigkeit zu entfernen. Der weitere Gang der Analyse bestand nun darin, dass die Flüssigkeit mit verdünnter Schwefelsäure im geringen Ueberschuss angesäuert wurde, um allen an die Chinasäuren gebundenen und gelösten Kalk aus der spirituösen Lösung als Sulfat zu fällen. Das gebildete Calciumsulfat wurde nun am folgenden Tage durch Filtriren entfernt und nach Auswaschen mit Spiritus von $92 \%$ die klare gelbgefärbte Alkaloïdlösung aus dem Wasserbade bis auf $10 \mathrm{~g}$. abdestillirt, zum Rückstande $50 \mathrm{~g}$. Wasser gesetzt und das Ganze bis zu starksaurer Reaction mit verdünnter Schwefelsäure versetyt. Nach der Entfernung der ausgeschiedenen Chinasäure und des Pflanzenfetts am folgenden Tage durch Filtration und nach gutem Auswaschen mit $\pm 50 \mathrm{Ccm}$. Wasser, wurde die klare saure Lösung auf dem Wasserbade zu $50 \mathrm{Ccm}$. eingeengt und darauf vorsichtig mit verdiunntem Ammoniak neutralisirt.

Bevor der Neutralisationspunkt erreicht wurde, krystallisirte bei chinareichen Rinden das Sulfat, so charakteristisch 
durch seine Krystalliorm, während der Neutralisation bereits theilweise aus. Das letztere wurde nach 12 stündigem Stehenlassen bei $+15^{\circ} \mathrm{C}$. auf einem kleinen Filtrum gesammelt und mit wenig Wasser abgewaschen, dann durch leisen Druck vorsichtig rom Wasser befreit, vom Filter entfernt, im Was. serbade bis zu constantem Gewichte ausgetrocknet und nach einstündigem Abkühlen an der Luft gewogen. Man bekomm so ein constantes Chininsulfat mit $7 \mathrm{Mol} . \mathrm{H}^{2} \mathrm{O}$ und erhält, indem man $1 / 760$ in der Mutterlange in Lösung gebliebenes Sulfat hinzurechnet und die erhaltene Zahl mit 0,289 multiplicirt, das in der untersuchten Rindenquantität enthaltene Chinin. Die nach Abscheidung des Chininsulfats zurückbleibende Mutterlauge wurde nun aul 30 oder $20 \mathrm{Ccm}$. eingedampft, mit Seignettesalz auf Cinchonidin und mit Jodkalium auf Chinidin untersucht, welche Alkaloüde jedoch in den boli. vianischen Rinden nur sporadisch vorkommen, schliesslich mit Natronlauge im Ueberschuss gefällt und nach 12 stündigem Stehenlassen das Präcipitat gesammelt, ausgewaschen und bis zu constantem Gewichte getrocknet. Das so erhaltene Gemenge von Cinchonin und amorphem Alkaloïde wurde sodann nach dem als das zweckmässigste constatirten Scheidungsverfahren von Moens mit Spiritus von $40 \%$, auf jedes Gramm des Alkaloïdgemenges $12 \mathrm{Ccm}$, behandelt. Man erlält beim Aufgiessen des Spiritus meistens anfänglich eine teigartige Masse, die bei längerem Verreiben und Kneten pulverförmig wird. Die resultirendo Lösung des amorphen Alkaloïds wurde nun auf dem Wasserbade abgedampft und zu constantem Gewicht gebracht und die erhaltene Gewichtsmenge in der Weise corrigirt, dass für jedes $\mathrm{Ccm}$. des gebrauchten Spiritus $1,5 \mathrm{Mgm}$. in Lösung gebrachtes Cinchonin in Rechnung gestellt wurde.

Schied sich bei der Untersuchung kein Chininsulfat aus, weil die untersuchte Rinde entweder kein oder nur äusserst wenig Chinin enthielt, so wurde die Lösung zu $30 \mathrm{Ccm}$. eingeengt und dann die Analyse in der angegebenen $W$ cise fortgesetzt, jedoch mit der Vorsorge, dass das etwa abgeschiedene Tartrat durch Auflösen in Salzsäure und Ausschüt- 
W. Stoeder, Alkaloïdbestimmung der Bolivia-Chinarinden ctc. 247 teln mit Natriumhydrat und Aether auf einen etwaigen Chiningehalt untersucht wurde.

Das im Vorstehenden geschilderte Verfahren giebt bei amerikanischen Chinarinden, welche blos Chinin, Cinchonin und amorphes Alkaloïd enthalten, Resultate, welche an Genauigkeit nichts zu wünschen übrig lassen. Hat man es aber auch mit Chinidin und Cinchonidin zu thun, so ist die Abscheidung des Chininsulfats nicht ohne grosse Beschwerden, da die Lösungsverhältnisse dieser Alkalö̈de durchaus nicht so differiren, wie die des Chinins und Cinchonins (Chivinsulfat $=1 / 760$, Chinidinsulfat $=1 / 350$, Cinchonidinsulfat $=1 / 13_{0}$, Cinchoninsulfat $=1 / 54$.) Die Reinheit der abgeschiedenen Alkaloïde wurde stets einer genaneren Controle unterworfen, indem z. B. das auskrystallisirte Chininsulfat aufgelöst und mit $\mathrm{NH}^{3}$ und Aether ausgeschïttelt wurde, oder indem das Chinin aus den später wieder vereinigten Alkaloïden als Tartrat gefällt und nun in der angegebenen Woise geprüft wurde, oder das isolirte Cinchonin mit Natriumhydrat gelöst und mit Aether behandelt wurde, wobei sich häufig noch lileine Mengen amorphes Alkaloïd fanden.

Die Bestimmung des Wassergehalts iil den untersuchten Chinarinden geschah durch Anstellung verschiedener Proben, wobei $1 \mathrm{~g}$. 2 Std. lang einem trocknen Luftstrome von 120 bis $140^{\circ}$ ausgesetzt wurden. Im Durchschnitte betrug derselbe $12,2 \%$.

Die hier folgenden Angaben über den Alkaloïdgehalt beziehen sich auf luftrockne Rinden, nicht auf absolut trockne, was unseres Erachtens bei Alkaloïdbestimmungen in Chinarinden den Vorzug verdient. Man kauft nie absolut trockne Rinden ein und bei Beurtheilung des Werthes einer China fragt man nicht nach dieser Zahl, sondern nach dem Procentgehalte der lufttrocknen Handelswaare. Es ist das ein Fehler, welcher bei der Aufstellung der holländischen Culturchina-Listen gemacht wird, welche für Handelszwecke weit mehr Bedeutung haben würden, wenn die Zahlen sich auf lufttrockne Rinden bezögen. Man betrachtet die darin gemachten Angaben als Werthziffern, was sie doch eigentlich 
248 W. Stoeder, Alkaloülbostimmung der Bolivia-Chinarinden etc.

nicht sind; zu solchen werden sie erst, wenn man sie mit 0,86 , entsprechend einem Wassergehalte von $13,5 \%$, multiplicirt, wodurch z. B. eine Gehaltzahl von $6 \%$ auf $5,1 \%$ reducirt wird. Will man aus den hier mitgetheilten Zahlen für lufttrockne Rinde diejenigen für absolut trockne Rinde berechnen, so mïssen dieselben mit 0,878 dividirt werden, wodurch ein Procentgehalt von 6 sich auf 6,8 erhöht.

Es folgen nun die Resultate für die einzelnen bolivianischen Sorten, woran sich eine tabellarische Uebersicht der gesammten Resultate schliesst:

1. und 2. Platte Calis a y - Chinarinde (Quinquinas Calisaya plats). - Diese Proben stellen die frühere Monopolchina vor, wie sie vor Ausrottung der Bäume in dem Handel vorkamen. Zur Zeit ist von dieser Sorte nichts mehr vorhanden; die ausgestellten Platten sind, was Schönheit und Gehalt anlangt, einzig in ihrer Art und vielleicht der letzte Rest aus der Monopolperiode $(1845$ - 1855.)

Alkalö̈dgehalt: Chinin $3,100 \%$, Cinchonin $0,490 \%$, amorphes Alkaloïd 0,195\%; Summa der Alkaloïde 3,795\%.

3,110 Chinin entsprechen 4,174 Sulfat mit $7 \mathrm{H}^{2} \mathrm{O}$. Diese Analyse ist aus 3 ausserordentlich wenig differirenden Bestimmungen gewonnen.

3. und 4. Platte Calis a y-Chinarinde (Quinquinas Calisaya plats), Platten, welche von Bäumen abstammen, welche nach 1874 und 75 in der Provinz Yungas entdeckt wurden, „sehr selten und sehr reich an Salzen " (Schuhkraft.)

Alkalö̈dgehalt: Chinin 4,268\%, Cinchonin $0,496 \%$, amorphes Alkaloïd 0,164\%; Summa der Alkaloïde 4,828\%. 4,268 Chinin $=5,728$ Sulfat.

Bei dieser Analyse wurde das Chinin auch als Tartrat bestimmt.

5. und 6. Platte Calisaya-Chinarinde mit der Epidermis (Qinquinas-Calisaya plats à épiderme). „Platten, abstammendiaus der Provinz Yungas, mit der Aussenrinde bekleidet; wahrscheinlich sehr alkaloïdreich." (Schuhkraft.)

Alkaloïdgehalt: Chinin 1,724\%, Cinchonin 0,468\%, amorphes Alkaloïd 0,172\%; Summa der Alkaloïde 2,364\%. 
W. Stueder, Alkaloüdbestimnung der Bolivia-Chinarinten ete. 249

7. Platte Calisay a-Chinarinde (Quinquinas Calisaya plats). „Platten von Bäumen in der Provinz Inquisivi stammend. Von dieser Baumart, welche wegen ihres geringen Gehalts an Salzen früher nicht gefällt wurde, finden sich noch zahlreiche Gruppen im Departement Cochabamba, die aber allmählig exploitirt werden und verschwinden, so dass auch hiervon allgemeine Ausrottung zu befürchten steht; die ausgestellten Platten sind ausgesuchte Exemplare, vielleicht $1-2 \%$ Salze enthaltend, aber im Ganzen ist die Rinde sehr arm, obschon zur Bereitung von Chinawein benutzbar."

Dic Analyse ergab ein besseres Resultat als erwartet war, nämlich: Chinin 3,292\%, Cinchonin 0,772\%, amorphes Alkaloïd $0,272 \%$; Summa der Alkaloide 4,336\%.

Die Rinde enthielt somit mehr Chinin als die Monopolchina.

8. Platte Chinarinde (Quinquinas plats). „Rinde, abstammend von Baumgruppen, welche in der neuesten Zeit in Espiritu Santo in der Provinz Yurucares entdeckt wurden, wahrscheinlich keine Spur von Chinin enthaltend, aber zu Chinawein brauchbar." (Schuhkraft.)

Die Abwesenheit des Chinins bestätigte die Analyse, welche $1,288 \%$ Cinchonin und $0,236 \%$ amorphes Alkaloïd, somit im Ganzen 1,524\% Alkalö̈de darin nachwies.

9. und 10. Platte Chinarinde. „Rinde von Bäumen aus der Provinz Larecuja und der Umgebung von Songo und Challaux, in Bolivia unter dem Namen Cascarilla Naranjada (orangefarbige China) bekannt, angeblich kein Chinin enthaltend, aber sehr reich an Cinchonin und zu Chinawein brauchbar." (Schuhkraft.)

Alkaloüdgehalt: Cinchonidin 1,516\%, Cinchonin 2,096\%, amorphes Alkaloïd 1,088\%; Summa der Alkaloïde 4,700\%.

Das Cinchonidin wurde als Tartrat abgeschieden und die Reinheit desselben durch Schütteln mit Aether constatirt.

11. Röhrenchina (Quinquinas roulés). „Zweigrinden von den Naranjadabäumen aus Songo und Challaux (9 und 10), werthlos und betrügerischerweise unter die feinen RöhrenCalisaya gemengt." (Schuhkraft.) 
250 W. Stoeder, Alkulödbestimmung der Bolivia-Chinarinden etc.

Alkaloïdgehalt: Cinchonin 2,044\%, amorphes Alkaloüd $0,632 \%$; Summe der Alkalöde 2,676\%. Cinchonidin war nur in Spuren nachweisbar.

12. und 13. Röhren-Calisaya-China (Quinquinas Calisaya roulés). „Sehr reiche Rinden, abstammend ron guten Calisayabäumen und wohl von jungen Stämmen, Zweigen und Schösslingen aus den im Erdboden gebliebenen alten Baumstämmen, wahrscheinlich $4 \%$ Chinin enthaltend; sic werden jährlich seltener und steht totale Ausrottung zu gewärtigen." (Schuhkraft.)

Alkaloidgehalt: Chinin 3,892\%, Cinchonin 0,564\%, amorphes Alkaloïd 0,144\%; Summa der Alkaloïde 4,60\%.

14-18. Röhrenchina (Quinquinas roulés). „Rinden von jungen Stämmen und Schösslingen aus der Provinz Caupolican und den Districten von Pelechuco und Apolobamba; sehr arm an Salzen." (Schuhkraft.)

Alkaloïdgehalt: Cinchonin $0,234 \%$, amorphes Alkaloïd $0,036 \%$; Summa der Alkaloïde $0,270 \%$.

19-23. Röhrenchina (Quinquinas roulés). „Rinden von den Zweigen der unter 7 beschriebenen Bäume; für den Handel werthlos, werden sie doch stets gesammelt und von den einheimischen Chinasammlern betrügerischerweise mit besseren Sorten gemengt." (Schuhkraft.)

Alkaloïdgehalt: Chinin $0,292 \%$, Cinchonin $0,005 \%$, amorphes Alkaloïd $0,184 \%$; Summa der Alkalöide $0,531 \%$.

Bei dieser Analyse war die Scheidung von Cinchonin und amorphem Alkaloï in der Weise, dass das Cinchonin sich pulverförmig abschied, nicht möglich; Lösen in Säure und Ausschüteln mit HNAO und Aether hatte besseres Resultat.

24. und 25. Röhrenchinarinde (Quinquinas roulés). „Zweigrinden von Bäumen aus dor Provinz Yungas und dem Districte von Chorobamba, soweit solche noch nicht gefällt sind; diese Sorte enthält höchstens $1 / 2 \%$ Chinin und wird von den Eingeborenen Quina verde genannt, in grosser Menge geschält und Handelsunkundigen statt guter roulés verkauft, womit die Rinde einige Aehnlichkeit hat." (Schuhkraft.) 
W. Stoeder, Alkaloüdbestimmung der Bolivia-Chinarinden ete. 251

Alkaloïdgehalt: Cinchonin 1,184, amorphes Alkaloïd 0,652; Summa der Alkaloïde $1,836 \%$.

26. und 27. Platte und gerollte Chinarinde: „Muster von Platten und Röhren, kürzlich aus Cohabamba importirt und von Baumgruppen aus der Provinz Vallegrande, etwa 50 Leguas westlich von Santa Crux, welche man auszubeuten beabsichtigt, wahrscheinlich nicht chininhaltig." (Schuhkraft.)

Alkalö̈dgehalt: Cinchonin 2,128\%, amorphes Alkaloïd $0,408 \%$; Summe der Alkaloïde 2,536\%.

27. Falsche Chinarinde (Faux bois). "Rinde der Baumart, welche die Aymaraindianer Car-hua Car-hua nennen und vielfach betriiglich unter die guten Sorten mengen (caru heisst bitter, hua $=\mathrm{d}$ i, die Wiederholung eines Wortes erhöht in der Aymarasprache seine Bedeutung, also sehr bitter)." (Schuhkraft.)

Alkaloïdgehalt: Chinin $0,308 \%$, Cinchonidin 0,512\%, Cinchonin $0,760 \%$, amorphes Alkaloïd $0,088 \%$; Summa der Alkaloïde $1,668 \%$.

Die empfangene Probe dieser Nummer schien ein Gemenge mehrerer Rinden zu sein, von denen der grösste Theil nicht das Aussehn von Chinarinden hatte, während einzelne Röhren an echte, jedoch junge Calisayarinde denken liessen. Hierfür spricht auch das erhaltene Resultat der Analyse.

Uebersicht der vorhergehenden Alkaloidbestimmungen in luftrockener Rinde. Wassergehalt $12,2 \%$.

\begin{tabular}{|c|c|c|c|c|c|c|c|c|c|c|c|c|c|}
\hline $\begin{array}{l}\text { Alkaloide } \\
\text { in } \% .\end{array}$ & $\begin{array}{l}\text { No. } \\
1,2\end{array}$ & 3,4 & 5,6 & 7 & 8 & 9,10 & 11 & 12,13 & $14-18$ & $19-23$ & $, 24,25$ & 26 & 27 \\
\hline Chinin & 3,110 & 4,268 & 1,724 & 3,292 & - & - & - & 3,892 & - & 0,292 & 一 & $\rightarrow$ & 00,308 \\
\hline Chinidin & - & - & - & 1 & - & - & - & - & - & - & - & - & - \\
\hline Cinchonidin & - & - & - & - & - & 1,516 & - & - & - & - & - & - & 0,512 \\
\hline Cinchonin & 0,490 & 0,496 & 0,468 & 0,772 & 1,288 & 2,096 & 2,044 & 0,564 & 0,234 & 0,055 & 1,184 & 2,128 & 0,760 \\
\hline Amorphes & & & & & & & & & & & & & \\
\hline Alkaloïd . & 0,195 & 0,164 & 0,172 & 0,272 & 0,236 & 1,088 & 0,632 & 0,144 & 0,036 & 0,184 & 0,652 & 0,408 & 0,088 \\
\hline $\begin{array}{l}\text { Summe der } \\
\text { Alkaloïde }\end{array}$ & 3,795 & 4,928 & 2,364 & 4,336 & 1,524 & 4,700 & 2,676 & 4,600 & 0,270 & 0.531 & 1,836 & 2,536 & 1,668 \\
\hline & & & & & & & & & & & & & \\
\hline
\end{tabular}


Man erkennt aus dieser Tabelle, dass die auf äusseren Kennzeichen begründete Werthbestimmung der Chinarinden im Allgemeinen mit den Resultaten der chemischen Analyse ziemlich gut übereinstimmt. Nur bei No, 5 und 6 ist die Angabe "sehr reich an Salzen" etwas anffallend gegenüber No. 3 und 4 mit ihrem hohen Chiningehalte.

Die Probe von No. 9 und 10 liess durch die Farbe del Innenfläche an Java succirubra denken, wofür auch die analytischen Resultate eine gewisse Analogie verrathen.

Man sielit, wie die Tabelle zeigt, dass 5 ausgezeichneten Sorten Calisaya 8 geringere Calisayaqualitäten gegenüberstehen und wenn man, ohne jetzt in Betracht zu ziehen, was dic Folge einer completen Ausrottung der Chinabäume werden kann, annimmt, was sicher geschehen kann, dass bei Ausfuhr aus Bolivia die echten Sorten das Minimum und die schlechten das Maximum der Masse bilden, dann hat man in der That ein Recht, die folgenden beiden Punkte zu betonen: erstens die dringende Nothwendigkeit, zum Gebrauche in der Medicin nur Chinarinden mit einem bestimnten Alkaloüdgehalte für jede Sorte zuzulassen und zweitens, was von noch viel grösserer Bedeutung ist, den ausgezeichneten Werth jeder Chinacultur, wo mit besonderer Sorgfalt sowohl der Anbau als die Sortirung und weitere Behandlnng bewacht wird, als den einzigen Weg, um die China in der That dasjenige leisten zu lassen, was sie als Arzneimittel leisten kann und muss.

\title{
Nachweis des Chloroforms und Vorkommen des Dextrins im Harn.
}

\author{
Von E. Reichardt.
}

Chloroform reducirt die Feh ling'sche Lösung und zwar ist diese Reaction eine höchst genaue, indem die kleinsten Mengen Chloroform bei genügend verdünnter Fehling'scher Lösung die Reduction nach wenigen Augenblicken Kochen 